\title{
An experimental methodology to capture user and gameplay data tied to cybersickness
}

THIAGO PORCINO, Universidade Federal Fluminense, Brazil

DANIELA TREVISAN, Universidade Federal Fluminense, Brazil

ESTEBAN CLUA, Universidade Federal Fluminense, Brazil

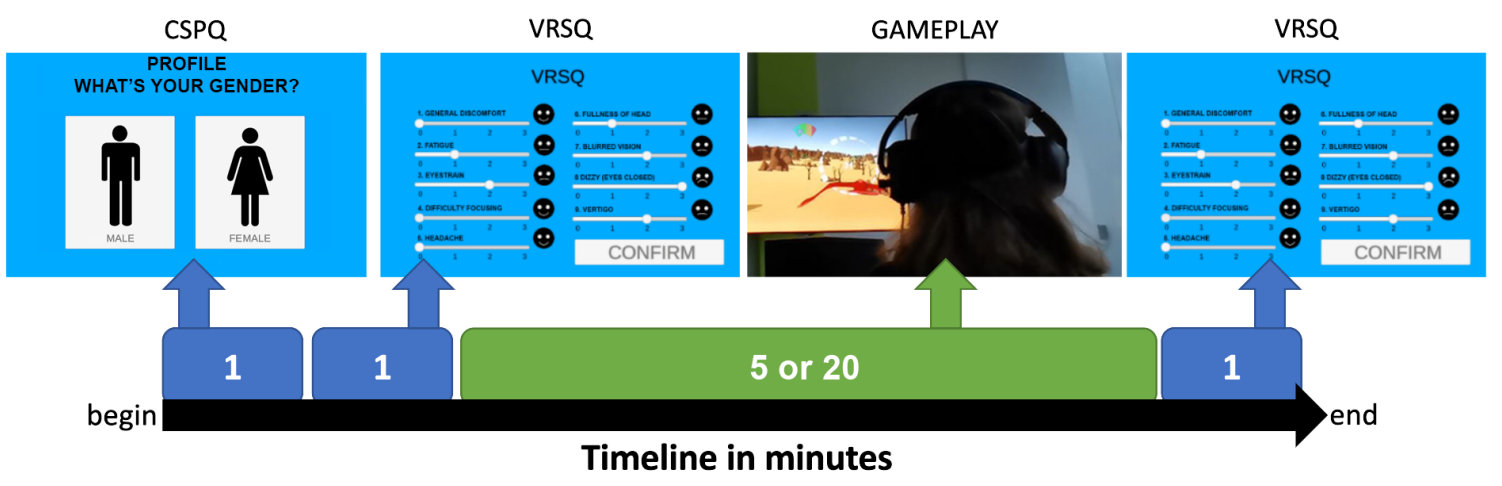

Fig. 1. The experience begins with the participants filling the Cybersickness Profile Questionnaire (CSPQ), followed by the VRSQ. Next, participants play the game for 5 (or 20) minutes. Finally, they fill a post-VRSQ questionnaire.

Virtual reality (VR) and head-mounted displays are constantly gaining popularity in various fields such as education, military, entertainment, and bio/medical informatics. Although such technologies provide a high sense of immersion, they can also trigger symptoms of discomfort. This condition is called cybersickness (CS) and is quite popular in recent publications in the virtual reality context. We created and conducted an iterative evaluating protocol methodology and proposed two VR games (a racing game and a flight game). The recorded data can be used for further machine learning analysis tied to cybersickness.

CCS Concepts: $\bullet$ Human-centered computing $\rightarrow$ Virtual reality.

Additional Key Words and Phrases: virtual reality, head mounted displays, cybersickness, data collection, games

\section{ACM Reference Format:}

Thiago Porcino, Daniela Trevisan, and Esteban Clua. . An experimental methodology to capture user and gameplay data tied to cybersickness. In XR in Games Workshop, together with IMX 2021: ACM International Conference on Interactive Media Experiences. June 21-23, 2021. NY, USA. ACM, New York, NY, USA, 5 pages.

\section{INTRODUCTION}

Head-mounted displays (HMDs) are one of the means of achieving immersive virtual reality environments. These devices usually consist of electronic displays and lenses that are fixed over the head where the display and lenses face

Published in accordance with the terms of the Creative Commons Attribution 4.0 International Public License (CC BY 4.0). Permission to reproduce or distribute this work, in part or in whole, verbatim, adapted, or remixed, is granted without fee, provided that the appropriate credits are given to the original work, not implying any endorsement by the authors or by SBC.2021 Brazilian Computing Society. 

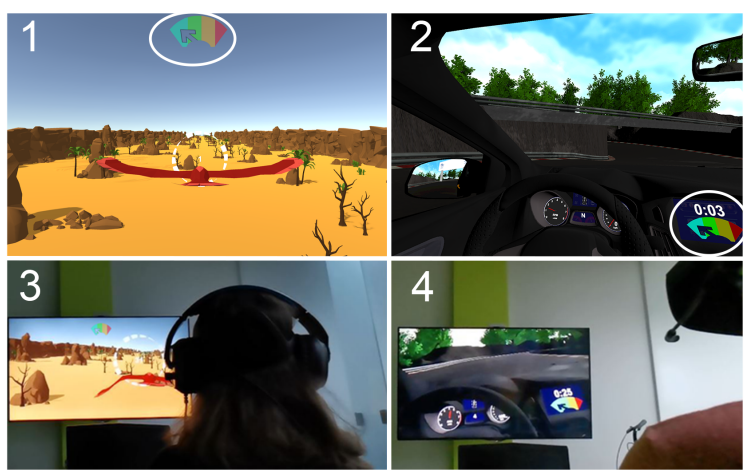

Fig. 2. Participants playing the flight game at the left ( 1 and 3$)$ and the race game at the right ( 2 and 4$)$. In images 1 and 2 , it is possible to see a visual feedback of their current discomfort level, marked with a circle.

the eyes of the user. HMDs are used for various purposes in the industry such as in games that focus on entertainment [12], military [11], education [1], therapy [3] and simulators for numerous contexts [7].

Unfortunately, HMDs are strongly related to frequent manifestations of discomfort [6]. Among the possible manifestations, cybersickness (CS) deserves special attention as it is the most frequent and is usually associated to long exposures to HMDs.

\section{MATERIALS AND METHODS}

Two different games were created for this work: (1) a race game and (2) a flight game as shown in Figure 2. In our protocol, we require the participants to fill in questionnaires before and after participating in a 5 or 20 minutes VR game session of our games.

In the race game interaction, the acceleration varies according to the choice of the user (they push the acceleration according to their will). In contrast, the flight game simulates an almost-constant acceleration. The player experience with both games is detailed in Figure 1.

The data collection occurred in a few different places such as schools, universities, and technological events. We spent a total of tree months collecting data with two HMD devices (HTC Vive and Oculus Rift). All participants agreed with their anonymous participation in the study and signed consent forms. The participants were allowed to quit the experiment whenever they wanted.

\subsection{Data visualization}

We build a data visualization application using Unity 3D. This application read our recorded dataset in CSV format and create graphical visualizations using position and discomfort attributes from data acquired in our protocols.

\section{GENDER DIFFERENCES ANALYSIS}

In this analysis, we used 3993 samples from race game recorded data. We observed that the occurrence of the discomfort reported by individuals occurs throughout the track (Figure 3) using position and discomfort level data presented in Table 1. However, the discomfort levels in specifics regions of track have a more significant accumulation. 


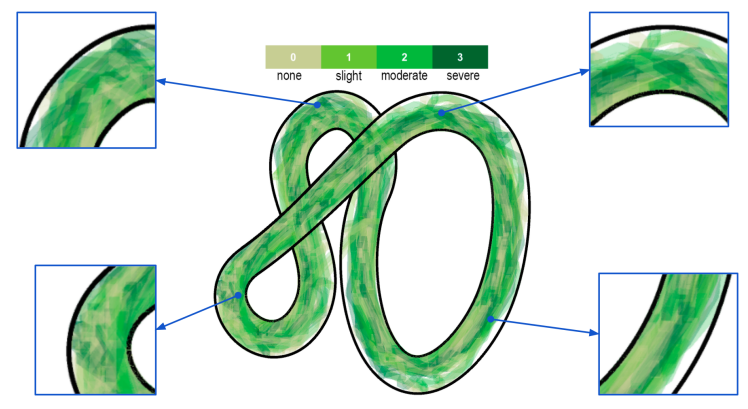

Fig. 3. Visualization of all moments where the participants of the elapsed game reported some of the levels of discomfort during the experiment. In the image, the intensity of discomfort reported by users varies from 0 (none) to 3 (severe) represented by the legend colors

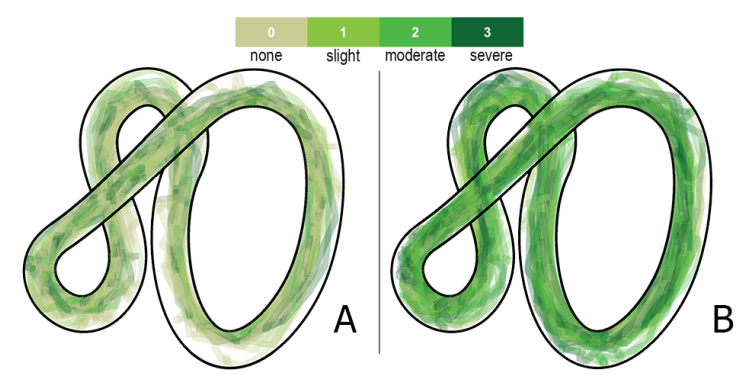

Fig. 4. comparative of the discomfort reported by female and male participants. In the image, the intensities of discomfort reported by users vary from 0 (none) to 3 (severe) represented by the legend colors.

In a comparative sample of reports of discomfort between individuals of the female gender (7 females with 1772 samples) and male (8 males with 2221 samples), We observed that in an accumulated result, the male participants reported discomfort values greater than zero more often than individuals of the female gender (illustrated in Figure 4).

Biocca [2] and Kolasinski [6], who report those female individuals are more susceptible to symptoms of MS. Despite being similar diseases, they have different environments and manifestations. Because of this, for this case, there is no way to say if there is a difference between genders for the manifestation of CS-based only on Biocca [2] and Kolasinski [6] works cited in this thesis. However, in this specific testing stage, the race track analysis results showed that the female audience reported less discomfort than the male audience.

Furthermore, we checked men participants performed the race game more competitively than women during the experiment, alternating acceleration shift frequency, and even crashing into track's corners. On the other hand, women played with near-constant acceleration shift frequencies avoiding collisions.

Additionally, Curry et al. [4] conducted a similar experiment. They examined (with SSQ) the influence of gender susceptibility and vehicle control on discomfort in two different VR experience tasks, where participants played (as vehicle driver) or viewed (as vehicle passenger) the game for up to 15 minutes. They also verified who had discontinued early in these experiences. Concerning MS incidences, the authors found no MS discrepancies among the participant's gender or groups (drivers and passengers). On the other hand, according to the authors, females participants discontinued early because of discomfort. In other words, the VR exposure time for female participants was significantly less than for 
Table 1. Raw feature set captured in Protocols 5 and 6, which contains numerical (N) and categorial (C) features.

\begin{tabular}{llll}
\hline \multicolumn{3}{c}{ Preliminary Feature set } \\
\hline \multicolumn{1}{c}{ Gameplay data } & \multicolumn{1}{c}{ Users data } \\
Feature & Type & Feature & Type \\
Time Stamp & N & Gender & C \\
Speed & N & Age & C \\
Acceleration & N & VR Experience & C \\
Rotation (x, y and z) & N & Flicker sensitivity & C \\
Position (x, y and z) & N & Pre-symptoms & C \\
Region Of Interest & C & Glasses wearing & C \\
Size of FOV & N & Vision Impairments & C \\
Frame Rate & N & Posture & C \\
Static Frame & C & Dominant Eye & C \\
Haptic Feedback & C & Discomfort Level & N \\
Degree of Control & C & & \\
DoF Simulation & C & & \\
Player Locomotion & C & & \\
Automatic Camera & C & & \\
\hline
\end{tabular}

male participants. Moreover, the authors were limited to 2 tasks and not deeply explored other virtual reality tasks associated with virtual reality discomfort.

According to recent studies [4, 5, 8], female individuals experienced better performance or magnified cognitive skills, localization, and picture tasks in VR than males. These results corroborate and suggest that gender differences in cybersickness may change besides different tasks in virtual reality environments.

Considering the gender attribute analysis we observed from the captured data of the race game (3993 samples, from 15 participants where seven are females and eight are males) that female individuals reported lower incidents of discomfort compared to male participants. This finding disagrees with literature in which Biocca [2] and Kolasinski [6] report that female individuals are more susceptible to symptoms of MS. However, such behavior was only observed in MS scenario and not in CS scenario. Anyways these findings need to be further investigated taking into account more samples and also with other games. Besides that, we were able to observe this effect only in the race game because in the flight game the gender data was not well distributed.

\section{CONCLUSION}

Conclusively, after applying these incremental and experimental protocols, we produce a 28 attributes dataset (see in Table 1) obtained from the following sources: profile data, questionnaire data, and gameplay data. All the features were captured considering two dependent variables: type of hardware and type of game. These attributes may works as a cybersickness database for machine learning analysis. The complete recorded data description are found in [10] and available in [9] for further analysis and reproducibility.

\section{REFERENCES}

[1] Kunjal Ahir, Kajal Govani, Rutvik Gajera, and Manan Shah. 2020. Application on virtual reality for enhanced education learning, military training and sports. Augmented Human Research 5, 1 (2020), 7.

[2] Frank Biocca. 1992. Will simulation sickness slow down the diffusion of virtual environment technology? Presence: Teleoperators \& Virtual Environments 1, 3 (1992), 334-343. 
[3] Mayra Carrión, Marco Santorum, Juan Benavides, Jose Aguilar, and Yolanda Ortiz. 2019. Developing a virtual reality serious game to recreational therapy using iPlus Methodology. In 2019 International Conference on Virtual Reality and Visualization (ICVRV). IEEE, $133-137$.

[4] Christopher Curry, Ruixuan Li, Nicolette Peterson, and Thomas A Stoffregen. 2020. Cybersickness in Virtual Reality Head-Mounted Displays: Examining the Influence of Sex Differences and Vehicle Control. International fournal of Human-Computer Interaction (2020), 1-7.

[5] Simone Grassini and Karin Laumann. 2020. Are modern head-mounted displays sexist? A systematic review on gender differences in HMD-mediated virtual reality. Frontiers in Psychology 11 (2020).

[6] Eugenia M Kolasinski. 1995. Simulator Sickness in Virtual Environments. Technical Report. DTIC Document.

[7] Uwe Kühnapfel, Hüseyin Kemâl Cakmak, and Heiko Maaß. 2000. Endoscopic surgery training using virtual reality and deformable tissue simulation. Computers \& graphics 24, 5 (2000), 671-682.

[8] Hai-Ning Liang, Feiyu Lu, Yuwei Shi, Vijayakumar Nanjappan, and Konstantinos Papangelis. 2019. Evaluating the effects of collaboration and competition in navigation tasks and spatial knowledge acquisition within virtual reality environments. Future Generation Computer Systems 95 (2019), 855-866.

[9] Thiago Porcino. [n.d.]. Cybersickness Dataset. https://github.com/tmp1986/UFFCSData. Accessed: 2021-04-03.

[10] Thiago Porcino, Erick O Rodrigues, Alexandre Silva, Esteban Clua, and Daniela Trevisan. 2020. Using the gameplay and user data to predict and identify causes of cybersickness manifestation in virtual reality games. In 2020 IEEE 8th International Conference on Serious Games and Applications for Health (SeGAH). IEEE, 1-8.

[11] Albert Rizzo, Thomas D Parsons, Belinda Lange, Patrick Kenny, John G Buckwalter, Barbara Rothbaum, JoAnn Difede, John Frazier, Brad Newman, Josh Williams, et al. 2011. Virtual reality goes to war: A brief review of the future of military behavioral healthcare. fournal of clinical psychology in medical settings 18, 2 (2011), 176-187.

[12] Bethesda Game Studios. 2015. The elder scrolls v: Skyrim. Bethesda Game Studios. 